\author{
Marquette University \\ e-Publications@Marquette
}

Biomedical Engineering Faculty Research and

Publications

Biomedical Engineering, Department of

$10-2011$

\title{
Motion Analysis of the Upper Extremities During Lofstrand Crutch- Assisted Gait in Children with Orthopaedic Disabilities
}

\author{
Brooke A. Slavens \\ Marquette University, brooke.slavens@marquette.edu \\ Neha Bhagchandani \\ Marquette University \\ Mei Wang \\ Marquette University, mei.wang@marquette.edu \\ Peter A. Smith \\ Shriners Hospital for Children \\ Gerald F. Harris \\ Marquette University, gerald.harris@marquette.edu
}

Follow this and additional works at: https://epublications.marquette.edu/bioengin_fac

Part of the Biomedical Engineering and Bioengineering Commons

\section{Recommended Citation}

Slavens, Brooke A.; Bhagchandani, Neha; Wang, Mei; Smith, Peter A.; and Harris, Gerald F., "Motion Analysis of the Upper Extremities During Lofstrand Crutch-Assisted Gait in Children with Orthopaedic Disabilities" (2011). Biomedical Engineering Faculty Research and Publications. 19.

https://epublications.marquette.edu/bioengin_fac/19 
Marquette University

e-Publications@Marquette

\section{Biomedical Engineering Faculty Research and Publications/College of} Engineering

This paper is NOT THE PUBLISHED VERSION; but the author's final, peer-reviewed manuscript. The published version may be accessed by following the link in the citation below.

Journal of Experimental \& Clinical Medicine, Vol. 3, No. 5 (October 2011): 218-227. DOI. This article is (C) Elsevier and permission has been granted for this version to appear in e-Publications@Marquette.

Elsevier does not grant permission for this article to be further copied/distributed or hosted elsewhere without the express permission from Elsevier.

\section{Motion Analysis of the Upper Extremities During Lofstrand Crutch-Assisted Gait in Children with Orthopaedic Disabilities}

Brooke A. Slavens

Department of Occupational Science and Technology, University of Wisconsin-Milwaukee, Milwaukee, WI

Shriners Hospital for Children, 2211 North Oak Park Avenue, Chicago, IL

Orthopaedic and Rehabilitation Engineering Center, 735 North 17th Street, Suite 105, Milwaukee, WI

Neha Bhagchandani

Department of Biomedical Engineering, Marquette University, P.O. Box 1881, Milwaukee, WI

Mei Wang

Department of Biomedical Engineering, Marquette University, P.O. Box 1881, Milwaukee, WI

Department of Orthopaedic Surgery, Medical College of Wisconsin, Milwaukee, WI

Orthopaedic and Rehabilitation Engineering Center, Milwaukee, WI

Peter A.Smith 
Shriners Hospital for Children, 2211 North Oak Park Avenue, Chicago, IL

\section{Gerald F.Harris}

Shriners Hospital for Children, 2211 North Oak Park Avenue, Chicago, IL

Department of Orthopaedic Surgery, Medical College of Wisconsin, Milwaukee, WI

Orthopaedic and Rehabilitation Engineering Center, Milwaukee, WI

\section{Abstract}

\section{Background}

This paper presents a review of current state-of-the-art dynamic systems for quantifying the kinematics and kinetics of the joints of the upper extremities during Lofstrand crutch-assisted gait. The reviewed systems focus on the rehabilitation of children and adults with myelomeningocele (MM), cerebral palsy (CP), spinal cord injury ( $\mathrm{SCl}$ ), and osteogenesis imperfecta (OI). Forearm crutch systems have evolved from models with single- to multi-sensor hardware systems that can incorporate an increasing number of segments that are in compliance with the standards of the International Society of Biomechanics (ISB).

\section{Methods}

The initial system developed by our group was a single, six-axis, sensor-crutch design with an accompanying ISBcompliant, inverse dynamics model. The model consisted of seven upper body segments and two crutch segments. After thorough validation of the software and hardware, it was tested using nine children with MM. The join dynamics of the shoulder, elbow, and wrist were assessed during reciprocal and swing-through gait.

\section{Results}

The dynamic metrics of the upper extremeties, including the mean, range, and maximum force and moment, were found to be significantly different depending on the gait pattern. Joint forces were found to be the greatest during swing-through gait, with inferior forces reaching $50 \%$ of body weight. In order to improve upon the initial system, our group developed a four-sensor crutch system that measures the contributions of the crutch-cuff kinetics. The inverse dynamics model was enhanced by including crutch-cuff and sensor segments that also follow the ISB modeling standards. This system was used to model subjects with $\mathrm{CP}$, SCl, and OI. Maximum joint forces were measured in the subject with $\mathrm{CP}$, while maximum moments were measured in the subject with $\mathrm{SCl}$. The subject with OI presented the smallest joint forces and moments.

\section{Discussion}

These novel model systems may be used to improve the quantification of joint dynamics during Lofstrand crutch-assisted gait. These methods may ultimately improve the identification of the risk factors for joint pathology and subsequent therapeutic planning and rehabilitation paradigms.

\section{Key words}

Biomechanics, inverse dynamics modeling, Lofstrand crutches, motion analysis, upper extremity

\section{Introduction}

Motion analysis has been extensively applied to the movements of the lower extremities during gait and now is being used to analyze the upper extremities (UEs) and the motion of the entire body. Complex challenges arise when quantifying the UEs because of their complicated movement patterns. Unlike the lower extremities, no standard movements exist, such as a repeating gait pattern. Due to the advanced movement patterns of the UE, inverse dynamics models are necessary to accurately capture their motions. In addition, with knowledge of UE 
dynamics, full body movements can be better understood. Evidence-based clinical questions are also driving up the demand for advanced UE models. Improved motion analysis technology, force sensor hardware, and modeling developments have allowed the growth of complex biomechanical models and associated hardware systems. These include systems for quantifying three-dimensional (3-D) UE joint dynamics during Lofstrand (forearm) crutch-assisted gait, which will be reviewed in this paper.

The latest National Institute on Disability and Rehabilitation Research (NIDRR) mobility device report estimates that there are over 6.8 million assistive device users, of which approximately 566,000 are crutch users. ${ }^{1}$ Among these, 36,000 are under 18 years of age. Extensive weight bearing due to long-term crutch usage in this pediatric population has been shown to lead to UE pain and pathologies such as shoulder arthropathy, arthritis, and carpel tunnel syndrome. ${ }^{2,3,4,5}$ The proposed risk factors for UE pain and pathology associated with the use of crutches include repetitive impulse loading and prolonged wrist extension and radial deviation. ${ }^{4}$ The literature has also reported that the use of Lofstrand crutches may lead to hand pain and sensory disturbances. ${ }^{4} \mathrm{~A}$ study conducted by Sie et al indicated that walking aids contribute to the development of shoulder pain. ${ }^{5}$ Accurate quantitative assessment is, therefore, essential for identifying the risk factors that lead to the development of pain and injury in long-term crutch users, as well as for developing appropriate rehabilitation strategies.

New advances in technology, standardized modeling methods, and greater access to patient populations has lead to recent investigations into the UE dynamics of crutch-assisted gait in persons with orthopaedic disabilities. Previously, analyses of the UE during mobility have been limited. ${ }^{6,7,8}$ The limitations of the reported studies on the dynamics of crutch-assisted gait have included nonstandardized kinematic models, joint angle computations, validation procedures, and small sample sizes. ${ }^{6,9,10}$ In addition, the scope of previous UE studies has not included pediatric applications., 10,11,12 Technological advancements now allow us to move beyond the simple study of joint motions to complex joint forces and the effects of mobility devices.

\section{Review of upper extremity inverse dynamics models of crutch-assisted gait}

Opila et al pioneered the study of the UE kinetics during gait by studying Lofstrand crutches and canes in 1987. ${ }^{3,13}$ Twelve subjects with three different pathologies-hip replacement, tibial fracture, and paraplegiawere investigated. The hardware system consisted of a six-axis strain gauge transducer placed on the shafts of each assistive device in order to measure the axial and shear forces that were applied to the aids. 3-D video analysis was used to measure the distance from the resultant crutch-force vector to the joint centers and subsequently compute the shoulder and elbow moments. Only device contact, not swing, was assessed. This study showed that the strengthening the specific components of the UE musculature is required to balance the moments of the loads of these assistive devices, thereby leading to more effective use.

Melis et al investigated the use of ambulatory-assistive devices in adults with incomplete spinal cord injury (SCl). ${ }^{9}$ The influence of canes, crutches, and walkers on assisted gait was addressed in order to better understand the interactions between the aids and the user. Seven reflective markers were placed on the whole body and three markers were placed on the device in order to capture their respective motions. Each device was installed with 16 strain gauges at the tip of each leg. The strain gauges were used to measure the orthogonal forces. Ten subjects with $\mathrm{SCl}$ were instructed to walk using a height-adjustable, instrumented, ambulatoryassistive device. Several parameters, including walking speed, cadence, step length, and the joint angles of the trunk, thigh, knee, and ankle were evaluated. Kinetic data, including axial compressive force, antero/posterior bending force, and medio/lateral bending force, were measured. The crutches were found to support up to $50 \%$ of the subject's body weight while providing lateral stability and antero/posterior restraint. It was concluded that rehabilitation specialists should match a device's specific characteristics to the user needs in order to prevent overuse injuries, especially those of the shoulder. 
Liggins et al investigated the motions and forces of crutches in the sagittal plane. ${ }^{14} \mathrm{~A}$ single six-axis load cell was placed at the tip of the crutch. The model used three reflective markers to define the rigid body segments of the crutch. Two subjects with incomplete $\mathrm{SCl}$ and cerebral palsy (CP) were used in this study. The loading patterns in the sagittal plane were unique to each subject. The subject with incomplete $\mathrm{SCl}$ demonstrated the largest forces. This study presented a preliminary UE biomechanical system that can be used to study the motions of crutches and their resulting ground reaction forces.

In order to accurately quantify crutch-assisted gait, a validated and standardized dynamic model must be applied. The Standardization and Terminology Committee of the International Society of Biomechanics (ISB) developed standards for UE modeling in $2005 .{ }^{15}$ This group proposed definitions that can be used to develop a joint coordinate system for studying the shoulder, elbow, and wrist. The adoption of these standards is encouraged by researchers and clinicians.

Requejo et al developed an inverse dynamics model and crutch system to investigate the UE kinematics and kinetics of forearm crutch-assisted gait in a single subject with $\mathrm{SCl}^{10}$ The model included six body segments and four crutch segments that were defined by 24 markers. The net joint forces and moments of the UE during crutch-assisted gait were characterized. Six-axis strain-gauge force sensors were placed below the crutch handle and near the forearm cuff in order to measure their reaction moments. The cuff forces were then derived from the cuff moments. The limitations of the study include the use of a nonstandardized model, indirect measurement of the forces involved, and small sample size. High superior forces were found at the wrist, elbow, and shoulder. This work presents a validated kinetic system for evaluating UE joint dynamics during crutchassisted gait.

Haubert and colleagues followed up this study by comparing 3-D shoulder joint reaction forces and stride characteristics during bilateral forearm crutch and front-wheeled walker ambulation. ${ }^{16}$ Reciprocal gait was investigated in adults with incomplete $\mathrm{SCl}$. The significant outcomes measured include the peak force, rate of loading, and force-time integral of each component of the net 3-D shoulder joint reaction force during ambulation with crutches and walkers. The largest weight-bearing force was superiorly directed, followed by the posterior force. The superior joint force demonstrated a significantly higher peak and rate of loading during crutch walking ( $48.9 \mathrm{~N}$ and $311.6 \mathrm{~N} / \mathrm{s}$, respectively, vs. $45.3 \mathrm{~N}$ and $199.8 \mathrm{~N} / \mathrm{s}$, respectively). ${ }^{16}$ The largest nonweight-bearing force was inferiorly directed with a significantly greater peak occurring during crutch ambulation (43.2 N vs. $23.6 \mathrm{~N}$ during walker gait, respectively). The subjects' walking velocities and cadences were similar. Stride lengths were significantly greater during crutch walking (62\% vs. $58 \%$ of normal). ${ }^{16}$ Shoulder joint moments and elbow and wrist joint reaction forces were not reported.

This paper presents further details regarding two UE inverse dynamics models of Lofstrand crutch-assisted gait and their applications to the pediatric population of crutch users. The Lofstrand crutch systems presented here have evolved from single- to multi-sensor designs. Both models are in compliance with the recommendations of the International Society of Biomechanics (ISB). ${ }^{15}$ The single-sensor design was studied using a set of children with myelomeningocele ( $\mathrm{MM}$ ) and used in conjunction with a custom, validated, inverse dynamics model. The multiple-sensor design was developed to account for the contributions of the crutch handle and forearm cuff in order to more fully quantify Lofstrand crutch-assisted gait. This model system was then applied to three children with $\mathrm{CP}, \mathrm{SCl}$, and $\mathrm{OI}$. These advanced inverse dynamics models and crutch systems may prove useful for the quantification of UE ambulation and the development of therapeutic gait strategies to help manage long-term crutch users. The investigation of the force demands placed on the UE may have a significant impact on rehabilitation protocols, injury prevention methods, and crutch designs. 


\section{Single-sensor Lofstrand crutch system}

\subsection{Methods}

To build upon our group's construction of a validated kinematic model of the UE in adult rehabilitation, ${ }^{17}$ we developed a pediatric model for assessing children with $\mathrm{MM} .{ }^{18}$ In this study, our group investigated reciprocal and swing-through crutch gait patterns.

\subsubsection{Kinematic model}

A 3-D biomechanical model was developed in accordance with the ISB recommendations. ${ }^{15}$ The UE model consisted of seven body segments: 1) thorax, 2) right upper arm, 3) right forearm, 4) right hand, 5) left upper arm, 6) left forearm, and 7) left hand (Figure 1). ${ }^{18,19}$ Two additional crutch segments (right and left) were also modeled. The rigid body segments were defined using 26 passive markers that were placed on bony anatomical landmarks and the crutches. The trunk and upper arm segments were connected by a shoulder joint with six degrees of freedom (i.e., glenohumeral joint). Two degrees of freedom, the elbow and wrist joints, were connected to the upper arm, forearm, and hand segments. Pronation/supination of the forearm was expressed in terms of the motion through the elbow, while varus/valgus was constrained. The joint centers were calculated using subject-specific anthropometric measurements. The joint coordinate systems also followed ISB conventions. ${ }^{15}$ The motion of the third metacarpal of the hand, with respect to the forearm, defined the global wrist motion.10, 15 Vicon BodyBuilder (version 3.6, Vicon, Oxford, England) was used to develop this model. The UE kinematic model was evaluated for accuracy and precision. ${ }^{17,19}$ Joint rotations were described using Euler angles (Z-X-Y sequence). ${ }^{20} \mathrm{~A} 14$-camera Vicon system captured the kinematic motions. The model kinematics and kinetic hardware were validated through rigorous analyses. ${ }^{19}$
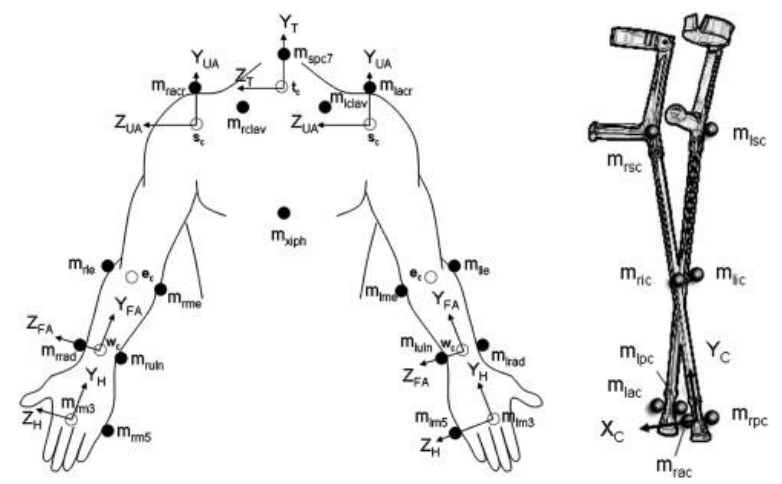

Figure 1. UE model marker placement, joint centers, and segmental coordinate systems. Right-handed coordinate systems were constructed following ISB conventions; the X-axis is directed anteriorly (abduction/adduction axis), the Y-axis is directed superiorly (internal/external rotation axis), and the Z-axis directed laterally to the right (flexion/extension axis). Markers are shown as black circles and joint centers are shown as open circles. Reprinted with permission from Elsevier. ${ }^{18}$

\subsubsection{Kinetic model}

A six-axis transducer (AMTI; Watertown, MA, USA) was mounted on the tip of each crutch in order to acquire the kinetic data. The force sensors measured the applied 3-D orthogonal reaction forces and moments during gait. The kinetic data were used to compute the tri-axial UE joint forces and moments. The contribution of the cuff forces and moments were assumed to be negligible and not included in this model.

\subsection{Results}

Following IRB approval, nine subjects ( $11 \pm 3.8$ years) with MM participated in this research study. Written parental and subject consent were obtained. All subjects had been diagnosed with L3- or L4-level 
myelodysplasia and were ambulatory using Lofstrand crutches. All subjects were recognized as having both reciprocal and swing-through gait patterns.

Ranges of motion and the mean joint forces and moments were calculated during gait. Kinematic and kinetic metrics were statistically analyzed by comparing reciprocal gait to swing-through gait using the Wilcoxon signed rank test.

All temporal and distance parameters were greater during swing-through gait in comparison with reciprocal gait, except duration of stance (Table 1). Significant differences between reciprocal and swing-through gait were found for stride length $(p=0.035)$ and stance duration $(p=0.016)$.

Table 1. Temporal and distance parameters measured during reciprocal and swing-through gait

\begin{tabular}{|l|l|l|l|l|}
\hline Gait pattern & $\begin{array}{l}\text { Cadence } \\
\text { (steps/min) }\end{array}$ & $\begin{array}{l}\text { Walking speed } \\
(\mathbf{m} / \mathbf{s})\end{array}$ & $\begin{array}{l}\text { Stride length } \\
(\mathbf{m})\end{array}$ & $\begin{array}{l}\text { Stance duration (\% gait } \\
\text { cycle) }\end{array}$ \\
\hline Reciprocal & 70 & 0.5 & 0.8 & 60 \\
\hline Swing-through & 77 & 0.7 & 1.0 & 50 \\
\hline
\end{tabular}

\subsubsection{Upper extremity kinematics}

The mean joint motions, for both the right and left sides, during reciprocal gait and swing-through gait patterns were determined (Figure 2). ${ }^{18}$ For both gait patterns, the joint angles of the thorax, shoulder, and elbow remain flexed throughout the gait cycle, while the wrist joint angles remain extended, although they had different motion pattern morphologies. The range of motion was greatest during swing-through gait. Reciprocal and swing-through gait patterns differed markedly with significant difference in terms of the ranges of the crutches, thorax, and elbow motions $(p<0.05)$.
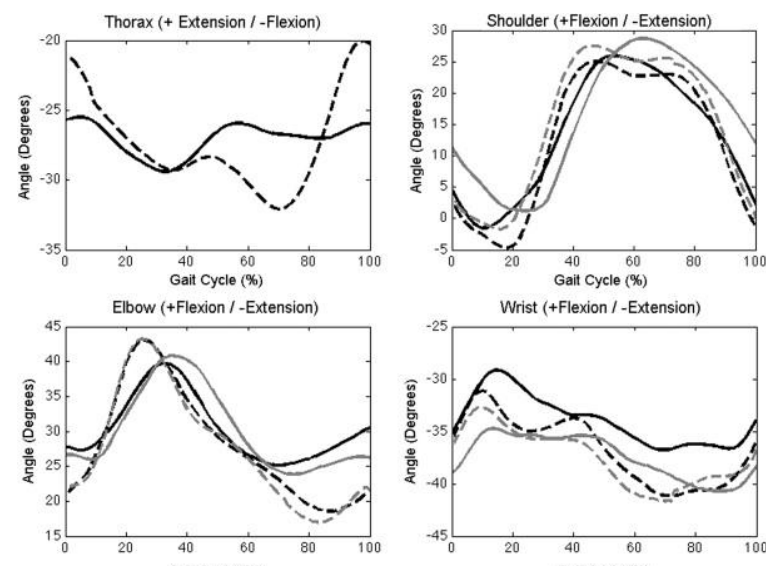

Gait Cycle (\%)

Figure 2. Mean UE kinematics (sagittal plane). The mean joint angles of the thorax, shoulders, elbows, wrists, and crutches are plotted during reciprocal gait (solid) and swing-through gait (dashed) through 0-100\% of the gait cycles. Right (black); left (gray). Reprinted with permission from Elsevier. ${ }^{18}$

\subsubsection{Upper extremity kinetics}

The mean peak joint forces were significantly different $(p<0.05)$ at all joints between reciprocal and swingthrough gait patterns (Figure 3$)^{18}$. Additional metrics, including the force-time integral, rate of joint loading, peak moments, and the percentages of the peak forces and moments that occurred in the gait cycle, were also investigated and found to be significantly different. 

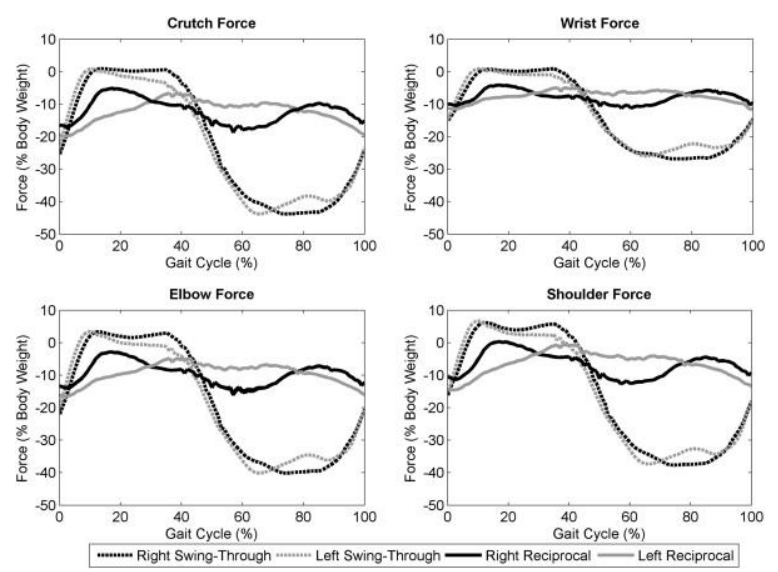

Figure 3. Mean joint forces of the right (black) and left (gray) crutches, wrists, elbows, and shoulders. Reciprocal gait (solid); swing-through gait (dashed). Superior force (+); inferior force (-). Reprinted with permission from Elsevier. ${ }^{18}$

\section{Multiple-sensor Lofstrand crutch system}

\subsection{Methods}

To expand on the previous work, our group developed an enhanced Lofstrand crutch system. ${ }^{21}$ This system included an ISB compliant inverse dynamics model and four-sensor crutch system that can incorporate crutch cuff kinetics in order to fully quantify the dynamics of the shoulder, elbow, and wrist. A study of this model using children with $\mathrm{CP}, \mathrm{SCl}$, and $\mathrm{Ol}$ is presented. The system may prove valuable for developing gait strategies for the management of long-term crutch users.

\subsubsection{Kinematic model}

The UE were modeled in a similar manner to the previously described inverse dynamics model, using seven rigid body segments and 18 markers. ${ }^{19}$ Each crutch was segmented into the cuff, handle, and lower crutch segments and defined by five markers. The model was developed using Vicon BodyBuilder.

\subsubsection{Kinetic model}

Each Lofstrand crutch was equipped with two six-axis force transducers (AMTI) in order to measure the reaction forces and moments at the crutch tip, handle, and cuff (Figure 4). ${ }^{21} \mathrm{~A}$ force transducer was positioned below the crutch handle to reduce the amount of inertial loading observed in previous model. A proximal force transducer was used to measure the tri-axial forces and moments at the forearm cuff. The transducers were positioned in order to allow adjustable crutch and cuff heights. The weight of the sensors $(0.1 \mathrm{~kg})$ was considerably less than those used in previous studies. ${ }^{10,16}$ The system underwent vigorous validation before use. ${ }^{21}$ 

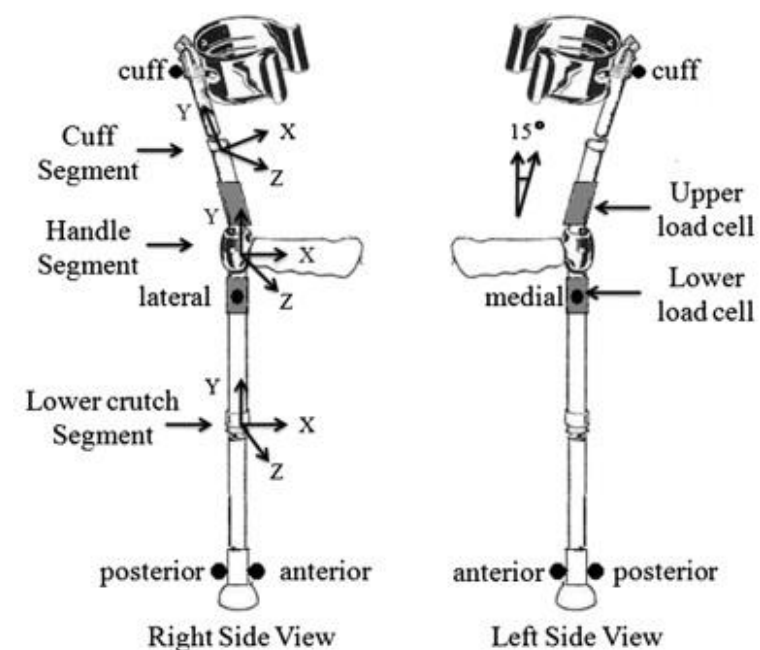

Figure 4. Crutch segment definitions and orientations of the axes. Each crutch segment consisted of a handle, lower crutch, and cuff segments. The cuff is offset from the lower crutch by a $15^{\circ}$ angle. Markers were placed on the cuff segment, right and left sides of the lower load cell, and on the anterior and posterior crutch tips.

Reprinted with permission from Elsevier. ${ }^{21}$

After IRB approval was granted and written parental and subject consent were obtained, as required by Shriners Hospital-Chicago, three children participated in this study (Table 2). The subject's disabilities included incomplete $\mathrm{SCI}$ (level T6), diplegic $\mathrm{CP}$, and type I OI.

Table 2. Subject-specific data

\begin{tabular}{|l|l|l|l|l|l|l|}
\hline Pathology & Age $(\mathbf{y})$ & Gender & Height $\mathbf{( m )}$ & Weight $\mathbf{( k g})$ & Handedness & Primary weight-bearing extremity \\
\hline $\mathrm{SCl}$ & 7 & Female & 1.2 & 20.4 & Right & Right \\
\hline $\mathrm{CP}$ & 11 & Female & 1.4 & 24.9 & Right & Left \\
\hline $\mathrm{OI}$ & 16 & Female & 1.4 & 43.8 & Right & Right \\
\hline
\end{tabular}

The subjects walked at a self-selected speed on a 6-m walkway while using the instrumented Lofstrand crutches. A 14-camera Vicon motion analysis system was used to capture the 3-D motions of each subject (120 Hz).

The joint kinetics were computed using the inverse dynamics Newton-Euler approach. ${ }^{22}$ Tri-planar motions of the thorax, shoulder, elbow, and wrist were evaluated and the range of motion of each joint was computed. Mean reaction forces and moments at the crutch tip, handle and cuff were calculated. Mean joint reaction forces and joint reaction moments were computed for the wrist, elbow, and shoulder; peak forces and moments were also assessed.

\subsection{Results}

Cadence, walking speed, stride length, and stance duration were analyzed (Table 3). Asymmetrical loading patterns were observed in all subjects. All subjects presented a pattern in which one extremity demonstrated notably higher forces and moments than the contralateral limb. For comparison among data, extremities were classified as primary or secondary weight-bearing extremities.

Table 3. Temporal and distance parameters

\begin{tabular}{|l|l|l|l|l|}
\hline Subject & Cadence (steps/min) & Walking speed (m/s) & Stride length (m) & Stance duration (\%) \\
\hline $\mathrm{SCl}$ & 97.8 & 0.5 & 0.6 & 68.6 \\
\hline
\end{tabular}




\begin{tabular}{|l|l|l|l|l|}
\hline $\mathrm{CP}$ & 78.3 & 0.6 & 1.0 & 56.3 \\
\hline $\mathrm{OI}$ & 103.4 & 0.8 & 0.9 & 65.4 \\
\hline
\end{tabular}

\subsubsection{Upper extremity kinematics}

\subsubsection{Crutch}

The subjects with $\mathrm{SCl}$ and $\mathrm{OI}$ tilted their crutches aft then forward in the sagittal plane, while the subject with $\mathrm{CP}$ tilted his crutches forward during the entire gait cycle (Figure 5). In the frontal plane, the crutch was laterally titled in all subjects. Crutch motion in the transverse plane was externally rotated in the subject with $\mathrm{SCl}$ and internally rotated in the subject with $\mathrm{OI}$. The subject with CP demonstrated internal crutch rotation from 0-40\% of the gait cycle, followed by external rotation.
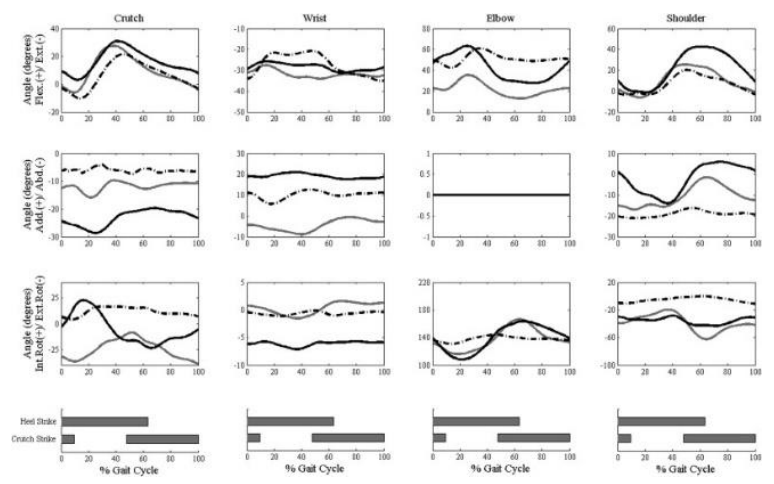

Figure 5. Mean crutch, wrist, elbow, and shoulder kinematics of the weight-bearing extremity. Subject with CP (black solid); subject with $\mathrm{SCl}$ (gray solid); subject with OI (black dashed dot).

The crutch motions of the secondary weight-bearing extremity demonstrated both fore and lateral tilt in all subjects. The crutch was also externally rotated by the subject with $\mathrm{SCl}$, while the subjects with $\mathrm{CP}$ and OI demonstrated internal and external rotation during the gait cycle.

The primary weight-bearing extremity presented greater range of motion (ROM) in subjects in the sagittal and frontal planes in patients with $\mathrm{SCl}$ and $\mathrm{CP}$. Similar ROM on both sides was demonstrated by the subject with OI. The ROM in the transverse plane was greater in the primary weight-bearing extremity in the subjects with $\mathrm{SCl}$ and $\mathrm{OI}$, while the subject with $\mathrm{CP}$ demonstrated greater ROM in the secondary weight-bearing extremity.

\subsubsection{Thorax}

All subjects presented flexion, lateral bending, and axial rotation of the thorax. The greatest ROM of the thorax was observed in the frontal plane. Maximum and minimum ROM were observed in the subjects with CP and OI, respectively.

\subsubsection{Shoulder}

Shoulder motion through the sagittal plane demonstrated extension through 0-30\% of gait cycle followed by flexion (Figure 5). The subject with $\mathrm{SCl}$ presented a different flexion morphology in the secondary weightbearing extremity throughout the entire gait cycle. For both sides, the shoulder was abducted and externally rotated throughout the gait cycle in all subjects. However, the subject with $\mathrm{CP}$ demonstrated adduction in the primary weight-bearing extremity through the second half of the gait cycle.

The subjects presented similar ROM of the shoulder through all planes of motion. The subject with CP demonstrated the largest ROM through the sagittal and coronal planes, while the subject with OI demonstrated the smallest ROM of the shoulder through all planes of motion on both sides. 


\subsubsection{Elbow}

The elbows displayed flexion and internal rotation during the entire gait cycle (Figure 5). The ROM of the elbow through the sagittal plane was greater in the secondary weight-bearing extremity compared with the primary weight-bearing extremity in the subjects with $\mathrm{CP}$ and OI. The elbow had the greatest ROM of all the UE joints studied, which was through the axial plane.

\subsubsection{Wrist}

Wrist kinematics demonstrated extension across all subjects on both sides (Figure 5). The wrist motion for the subjects with $\mathrm{CP}$ and $\mathrm{OI}$ demonstrated adduction (ulnar deviation), while the subject with $\mathrm{SCl}$ demonstrated abduction (radial deviation) throughout the gait cycle. On the secondary weight-bearing extremity, the wrist demonstrated extension, abduction (radial deviation), and external rotation. Of all the UE joints studied, the wrist demonstrated the smallest ROM.

\subsubsection{Upper extremity kinetics}

\subsubsection{Crutch tip}

The maximum crutch-tip force was in the inferior direction for both extremities among all subjects examined (Figure 6). The maximum forces were measured in the subject with CP.
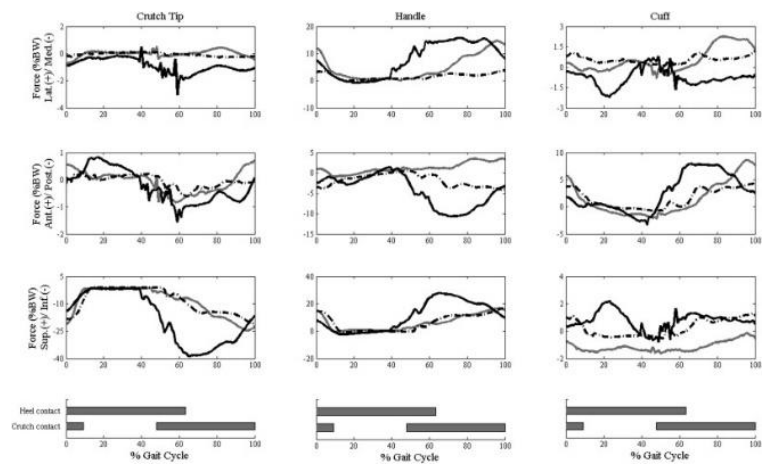

Figure 6. Crutch tip, crutch handle, and crutch cuff reaction forces of the primary weight-bearing extremity. Forces are normalized to percent body weight (\% BW). Subject with CP (black solid); subject with SCl (gray solid); subject with Ol (black dashed dot).

The greatest moments of the crutch tip were for lateral tilt (Figure 7). Maximum moments were demonstrated by the subject with $\mathrm{CP}$.
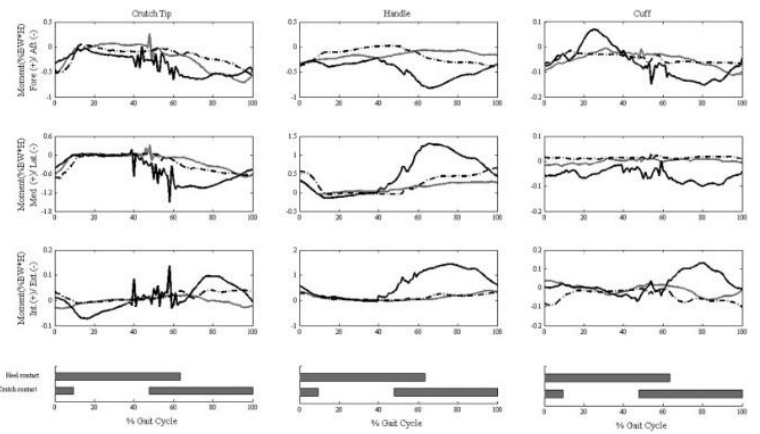

Figure 7. Crutch tip, crutch handle, and crutch cuff reaction moments of the primary weight-bearing extremity. Moments are normalized to percent body weight multiplied by height (\% BW*H). Subject with CP (black solid); subject with SCI (gray solid); subject with OI (black dashed dot). 


\subsubsection{Handle}

The maximum crutch handle forces were superiorly directed in both extremities and all subjects (Figure 6). The subject with $\mathrm{CP}$ demonstrated the greatest forces.

Aft tilt, medial tilt, and internal rotation moments were measured in both extremities and all subjects (Figure 7). The maximum handle moments were measured in the subject with $\mathrm{CP}$.

\subsubsection{Cuff}

The maximum cuff forces were measured in the anterior direction (Figure 6). The greatest forces were measured in the subject with $\mathrm{SCl}$. The greatest cuff moments were measured for internal rotation in the subject with $\mathrm{CP}$ (Figure 7).

\subsubsection{Wrist}

The wrist force was medial, posterior, and superior in all subjects (Figure 8). ${ }^{21}$ The subject with CP presented the maximum wrist force.
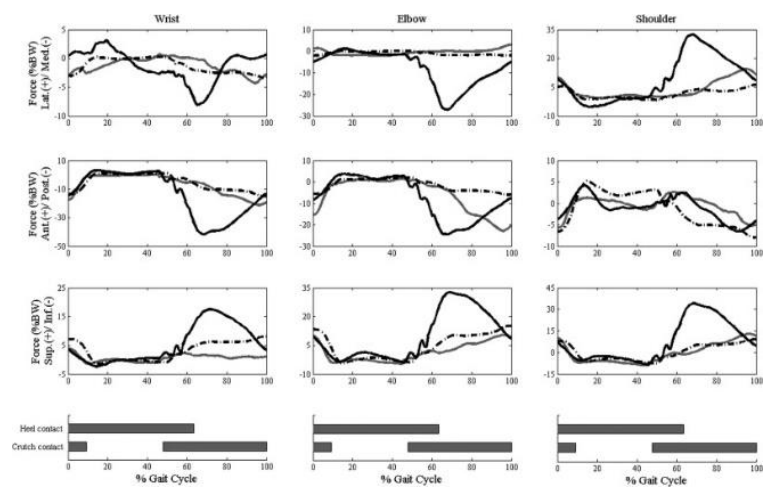

Figure 8. Wrist, elbow, and shoulder joint reaction forces of the primary weight-bearing extremity. Forces are normalized to percent body weight (\% BW). Subject with CP (black solid); subject with SCl (gray solid); subject with OI (black dashed-dot). Reprinted with permission from Elsevier. ${ }^{21}$

Flexion, adduction (ulnar deviation), and internal rotation moments were measured in all subjects through the gait cycle (Figure 9). The largest peak moments for the wrist were measured in the subject with CP.
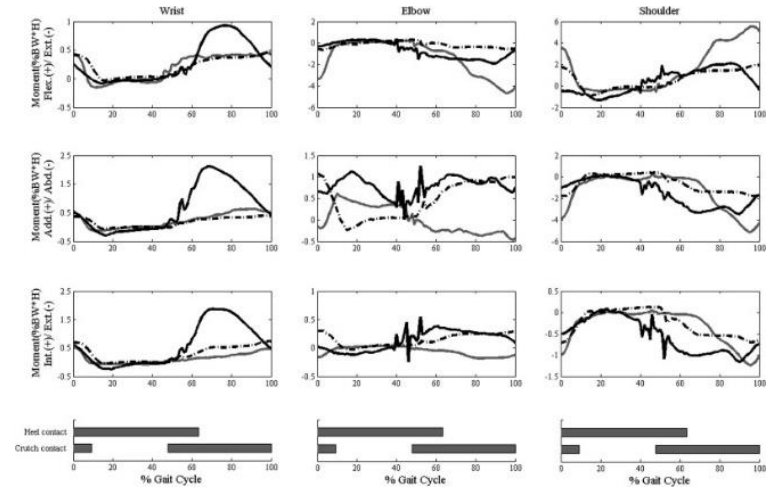

Figure 9. Wrist, elbow, and shoulder joint reaction moments of the primary weight-bearing extremity. Moments are normalized to percent body weight and multiplied by height $\left(\% \mathrm{BW}^{*} \mathrm{H}\right)$. Subject with $\mathrm{CP}$ (black solid); subject with $\mathrm{SCl}$ (gray solid); subject with OI (black dashed dot). Reprinted with permission from Elsevier. ${ }^{21}$ 


\subsubsection{Elbow}

High superior and posterior elbow forces were measured in all subjects (Figure 8). The subject with CP demonstrated the maximum elbow forces.

The extension moments were the greatest of the elbow moments (Figure 9). The subject with $\mathrm{SCl}$ demonstrated the maximum elbow moment.

\subsubsection{Shoulder}

The shoulder forces were both lateral and superior and moved between the posterior and anterior directions in all subjects (Figure 8). The maximum shoulder joint forces were measured in the subject with CP.

The flexion moments of the shoulder were greatest among the three planes measured (Figure 9). The subject with $\mathrm{SCl}$ demonstrated the maximum moments.

\section{Discussion}

The goal of these studies was to develop and apply state-of-the-art instrumented crutch systems to assess UE dynamics during Lofstrand crutch-assisted gait. Single- and multi-sensor systems were designed and applied to study several pediatric pathologies, including $\mathrm{MM}, \mathrm{SCl}, \mathrm{CP}$, and $\mathrm{OI}$.

Improvements in the design of this system occurred as the design shifted from a single- to multi-sensor system. The application of two six-axis transducers per crutch allowed the full characterization of joint kinetics by accounting for multiple crutch tip, handle, and cuff contributions that occur during loading. The location of the transducer below the handle reduced inertial loading effects. The additional force transducer placed near the cuff allowed the direct measurement of the cuff kinetics. Inclusion of this data into the kinetic model improved the accuracy of the forces and moments that were measured at the wrist, elbow, and shoulder.

The results of these studies support the use of technically validated crutch systems to evaluate UE ambulation patterns during Lofstrand crutch-assisted gait. Limitations of previous studies including the use of a limited number of sensors, poor sensor location, poor sensor characteristics, and the use of nonstandardized models have been addressed. ${ }^{9,10,13,16,19}$ The UE models used by Slavens et al were ISB compliant and include several design aspects that should be understood by researchers and clinicians.

Further insight could be obtained by conducting this research with a larger sample size in order to study additional gait patterns such as swing-through, swing-to, and three- and four-point reciprocal gait patterns. The current kinetic models allow for the detailed study of the demands of the joint loads during gait. How these forces and moments interact could be optimized through activity modification, gait training, and crutch redesign. Future research in this field may also offer valuable insight into crutch prescription, placement patterns, and injury- and pain-prevention strategies for long-term users of assistive devices.

\section{Conclusions}

Motion analysis can be used to study full body movements of the lower extremities and UEs due to new advances in inverse dynamics modeling and force sensor technology. These new methods have been applied to the study of clinical biomechanics in order to better understand orthopaedic pathologies such as $\mathrm{MM}, \mathrm{CP}, \mathrm{SCl}$, and OI. Due to the increased number of persons using assistive mobility devices, these new techniques can help to clarify the demands that joint loads place on the upper limbs. Several models have been developed to characterize Lofstrand crutch-assisted gait, allowing the evolution from single-sensor models to multi-sensor and multi-axis systems. Along with these advanced systems, inverse dynamics models continue to be developed with an increasing need for greater resolution, accuracy, and precision of UE joint kinetics. 3-D data of the 
shoulder, elbow, wrist, and crutch forces and moments allow clinicians and scientists to improve patient care in terms of reducing the long-term risk of developing UE pain and injury.

\section{Acknowledgments}

The authors would like to thank the teams at Shriners Hospital for Children-Chicago and the Orthopaedic and Rehabilitation Engineering Center (OREC) at Marquette University/The Medical College of Wisconsin. This work was also supported in part by NIDRR Advanced Rehabilitation Research Training (ARRT) Grant H133P080005, Dr. Ralph, and the Marian Falk Medical Trust.

\section{References}

1 H.S. Kaye, T. Kang, M.P. LaPlante. Mobility device use in the United States. U.S. dept. of education. disability statistics report. National Institute on Disability and Rehabilitation Research, Washington, D.C. (2000)

2 S. Lal. Premature degenerative shoulder changes in spinal cord injury patients. Spinal Cord, 36 (3) (1998), pp. 186-189

3 K.A. Opila, A.C. Nicol, J.P. Paul. Upper limb loadings of gait with crutches. J Biomech Eng, 109 (4) (1987), pp. $285-290$

4 D.A. Sala, L.M. Leva, F.J. Kummer, A.D. Grant. Crutch handle design: effect on palmar loads during ambulation. Arch Phys Med Rehabil, 79 (11) (1998), pp. 1473-1476

5 I.H. Sie, R.L. Waters, R.H. Adkins, H. Gellman. Upper extremity pain in the postrehabilitation spinal cord injured patient. Arch Phys Med Rehabil, 73 (1) (1992), pp. 44-48

6 A. Bartonek, H. Saraste, M. Eriksson, L. Knutson, A.G. Cresswell. Upper body movement during walking in children with lumbo-sacral myelomeningocele. Gait Posture, 15 (2) (2002), pp. 120-129

7 R.T. Gupta, S. Vankoski, R.A. Novak, L.S. Dias. Trunk kinematics and the influence on valgus knee stress in persons with high sacral level myelomeningocele. J Pediatr Orthop, 25 (1) (2005), pp. 89-94

8 T.C. Nguyen, R. Baker. Two methods of calculating thorax kinematics in children with myelomeningocele. Clin Biomech, 19 (10) (2004), pp. 1060-1065

9 E.H. Melis, R. Torres-Moreno, H. Barbeau, E.D. Lemaire. Analysis of assisted-gait characteristics in persons with incomplete spinal cord injury. Spinal Cord, 37 (6) (1999), pp. 430-439

10 P.S. Requejo, D.P. Wahl, E.L. Bontrager, C.J. Newsam, J.K. Gronley, S.J. Mulroy, J. Perry. Upper extremity kinetics during Lofstrand crutch-assisted gait. Med Eng Phys, 27 (1) (2005), pp. 19-29

11 R.A. Bachschmidt, G.F. Harris, G.G. Simoneau. Walker-assisted gait in rehabilitation: a study of biomechanics and instrumentation. IEEE Trans Rehabil Eng, 9 (1) (2001), pp. 96-105

12 G. Rab, K. Petuskey, A. Bagley. A method for determination of upper extremity kinematics. Gait Posture, 15 (2) (2002), pp. 113-119

13 K.A. Opila, A.C. Nicol, J.P. Paul. Forces and impulses during aided gait. Arch Phys Med Rehabil, 68 (10) (1987), pp. 715-722

14 The case for using instrumented crutches during gait analysis. In: Proceedings of the IEEE 28th Annual Northeast IEEE Bioengineering Conference. Philadelphia, PA; 2002.

15 G. Wu, F.C. van der Helm, H.E. Veeger, M. Makhsous, P. Van Roy, C. Anglin, J. Nagels, et al. ISB recommendation on definitions of joint coordinate systems of various joints for the reporting of human joint motion. Part II: shoulder, elbow, wrist and hand. J Biomech, 38 (5) (2005), pp. 981-992

16 L.L. Haubert, D.D. Gutierrez, C.J. Newsam, J.K. Gronley, S.J. Mulroy, J. Perry. A comparison of shoulder joint forces during ambulation with crutches versus a walker in persons with incomplete spinal cord injury. Arch Phys Med Rehabil, 87 (2006), pp. 63-70

17 B.A. Hingtgen, J.R. McGuire, M. Wang, G.F. Harris. An upper extremity kinematic model for evaluation of hemiparetic stroke. J Biomech, 39 (4) (2006), pp. 681-688

18 B.A. Slavens, P.F. Sturm, R. Bajorunaite, G.F. Harris. Upper extremity dynamics during Lofstrand crutchassisted gait in children with myelomeningocele. Gait Posture, 30 (2009), pp. 511-517 
19 B.A. Slavens, P.F. Sturm, G.F. Harris. Upper extremity inverse dynamics model for crutch-assisted gait assessment. J Biomech, 43 (10) (2010), pp. 2026-2031

20 G.F. Harris, P.A. Smith. Human motion analysis. IEEE Press, New York (1996)

21 B.A. Slavens, N. Bhagchandani, M. Wang, P.A. Smith, G.F. Harris. An upper extremity inverse dynamics model for pediatric Lofstrand crutch-assisted gait. J Biomech, 44 (2011), pp. 2162-2167

22 V.M. Zatsiorsky. Joint torques and forces: the inverse problem of dynamics. L.D. Robertson (Ed.), Kinetics of human motion, Human Kinetics, Champaign, IL (2002), pp. 365-454 